\title{
Simulation of future COVID-19 epidemic by vaccination coverage scenarios in Japan
}

\author{
Yuki Furuse \\ ${ }^{1}$ Institute for Frontier Life \\ and Medical Sciences, \\ Kyoto University, Kyoto, \\ Japan \\ ${ }^{2}$ Hakubi Center for \\ Advanced Research, \\ Kyoto University, Kyoto, \\ Japan \\ ${ }^{3}$ Nagasaki University \\ Graduate School of \\ Biomedical Sciences, \\ Nagasaki, Japan
}

\section{Correspondence to:}

Dr. Yuki Furuse Institute for Frontier Life and Medical Sciences, Kyoto University 53 Shogoin Kawaharacho, Sakyo-ku

Kyoto 606-8507 Japan

furusey.kyoto@gmail.com
Background COVID-19 continues to impose significant morbidity and mortality in Japan even after implementing the vaccination program. It would remain elusive if restrictions for its mitigation were to be lifted or relaxed in the future.

Methods A simulation study that explored possible vaccination coverage scenarios and changes in the intensity of nonpharmaceutical intervention restrictions was performed to assess the impact of COVID-19 based on death count.

Results Assuming the basic reproduction number of circulating viruses was 5.0, vaccines could prevent $90 \%$ of infections and $95 \%$ of deaths, and the vaccination coverage rate was high $(75 \%, 80 \%$, and $90 \%$ in people aged $12-39$ years, $40-59$ years, $\geq 60$ years, respectively), approximately 50000 deaths would occur over 150 days in Japan if all restrictions were lifted. Most deaths would occur among older adults, even if their vaccination coverage was assumed to be especially high. A low vaccination coverage scenario $(45 \%, 60 \%$, and $80 \%$ in people aged $12-39$ years, $40-59$ years, $\geq 60$ years, respectively) would require periodic implementation of strict measures even if the modified lifestyle observed in 2020 was sustained and vaccines were very effective. Some restrictions could be relaxed under high vaccination coverage. However, in the worst-case scenario where vaccines had decreased efficacy, as we have observed for the Delta variant, and people lived a relaxed lifestyle, our simulation suggests that even high vaccination coverage would occasionally require strict measures.

Conclusions We should carefully explore a manageable degree of restrictions and their relaxation. We will have to keep bracing for occasional surges of COVID-19 infection, which could lead to strict measures, such as those under a state of emergency. Such strategies are essential even after a wide rollout of vaccination.

COVID-19 caused a pandemic in 2020 and has affected many countries, including Japan. The first COVID-19 case in the country was confirmed on 15 January 2020 [1], and the cumulative numbers of cases and deaths reached 1 million ( 8 per 1000 population) and $15000(\sim 1$ per 10000 population), respectively, in August 2021. Nonpharmaceutical interventions (NPIs), such as physical distancing, wearing a face mask, rapid case detection, contact tracing, and isolation, play a significant role in controlling the COVID-19 epidemic [2,3]. When COVID-19 cases surged and concern about the collapse of health systems grew in Japan, a state of emergency was issued - asking people to stay at home and limit mass gatherings and asking businesses, including restaurants and bars, to reduce their hours or close. Although these measures were not mandatory but rather advisory, many citizens voluntarily followed them. As a result, the number of cases eventually decreased [4].

Several vaccines for COVID-19 were developed and became in use within one year after the emergence of SARS-CoV-2. In Japan, two vaccines, namely, BNT162b2 and mRNA-1273, were 
approved and administered to people $\geq 12$ years old. These vaccines reportedly prevent $90 \%$ of infections and $~ 95 \%$ of hospitalizations, severe illnesses, and deaths due to COVID-19 [5-10]. In Japan, vaccines were first administered to health care workers and those aged $\geq 65$ years and then to other populations. The vaccination coverage for the prioritized groups reached $~ 80 \%-90 \%$, and the number of COVID-19 cases among them dramatically dropped [11]. However, the vaccination coverage for the total population in Japan was still less than $50 \%$ as of August 2021 [12].

The Delta variant of SARS-CoV-2, which seems to have high transmissibility, was introduced and disseminated throughout Japan in June-August 2021, generating the fifth wave of the COVID-19 epidemic in the country [13]. While the basic reproduction number of the SARS-CoV-2 original strain was $2.5[14,15]$, the reproduction number of the Delta variant was estimated to be 5.0 [16]. In addition, the Delta variant reportedly decreases the efficacy of vaccines. Yet, vaccines still effectively prevent $~ 70 \%-90 \%$ of hospitalizations and fatal outcomes [17-20]. The pathogenicity of the Delta variant might have increased as well [21,22].

Whether a wide rollout of vaccination will control the spread of COVID-19 at a population level remains unknown. We thus performed a simulation study to assess the effect of vaccination on the impact of the disease by exploring several vaccination coverage scenarios in Japan. We also analyzed if and how NPI restrictions could be lifted or relaxed in the future.

\section{METHODS}

The spread of infection was simulated using a deterministic compartmental model with the following compartments: $S_{n}$, susceptible, unvaccinated; $S_{v}$, susceptible, vaccinated; $I_{n}$, infectious, unvaccinated; $I_{v}$, infectious, vaccinated; $C$, severe illness; $D$, death; $R$, recovered; and $V$, protected, vaccinated. The compartments were stratified into four age groups: 0-19, 20-39, 40-59, and $\geq 60$ years. The details of the model and parameters are described in Figure 1 and Table S1 in the Online Supplementary Document; the parameter values were based on empirical data from Japan.
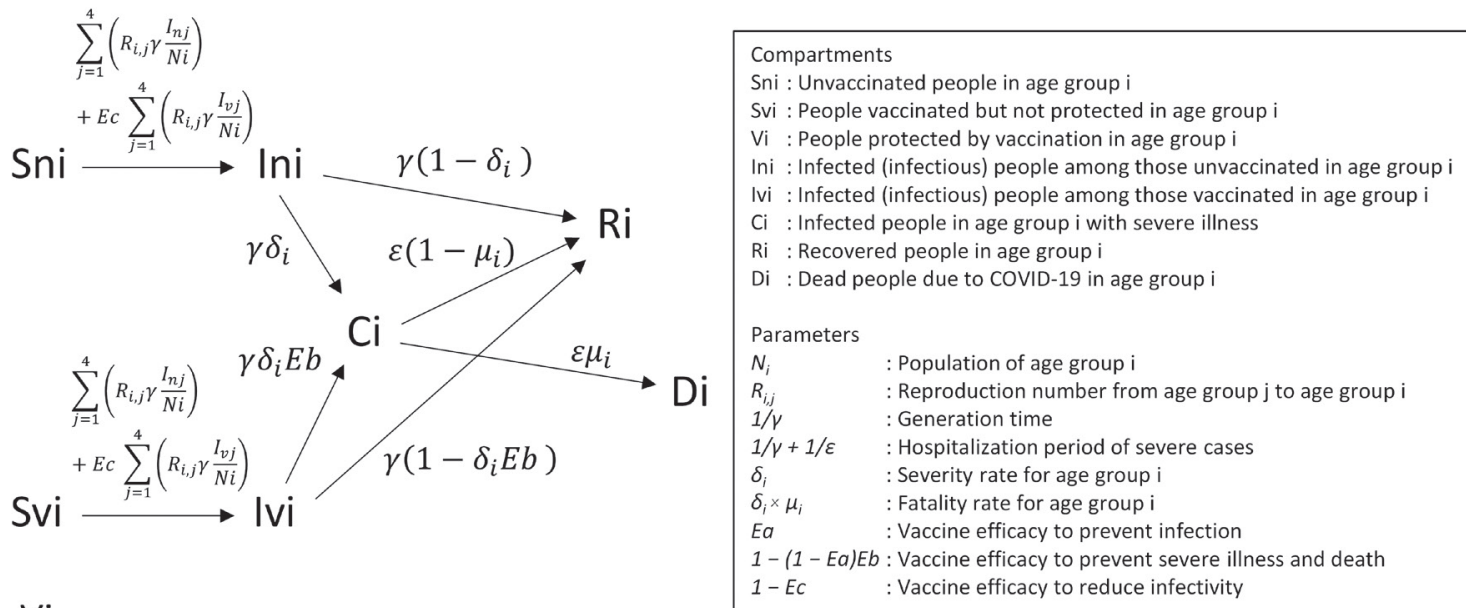

$\mathrm{Vi}$

Figure 1. Compartmental model for the simulation. The compartmental model for age group i is shown. The model consists of four age groups. Arrows and italic characters respectively depict transitions between compartments and their rates. The compartments and parameters in the model are explained in Table S1 in the Online Supplementary Document.

The reproduction number of circulating viruses and the efficacy of vaccines were subject to change, and five scenarios of vaccination coverage were tested in the simulation (Table 1 ). The assumptions for these vaccination coverage scenarios were based on a July 2021 survey in Japan [23]. It was assumed that vaccination could decrease the probability of infection, severe illness, and death [5-10,17-20], and it also could reduce infectivity from patients with a breakthrough infection [24-26].

The effectiveness of past NPIs in Japan was calculated from time-dependent effective reproduction numbers of COVID-19 (Figure S1 in the Online Supplementary Document). A 40\% reduction in transmission owing to NPIs was observed as a baseline in 2020. The transmission rate dropped by $70 \%$ according to the state of emergency order (see "Background" for details about the state of emergency in Japan). 
Table 1. Scenarios for the simulation*

\begin{tabular}{|c|c|c|}
\hline $\begin{array}{l}\text { Basic reproduction } \\
\text { number scenario }\end{array}$ & Value & Description \\
\hline & 2.5 & Corresponding to the original strain \\
\hline & 3.5 & Corresponding to the Alpha variant \\
\hline & 5.0 & Corresponding to the Delta variant \\
\hline & 7.5 & $\begin{array}{l}\text { Corresponding to } 50 \% \text { higher transmissibility than the current estimation } \\
\text { for the Delta variant }\end{array}$ \\
\hline $\begin{array}{l}\text { Vaccination coverage } \\
\text { scenario }\end{array}$ & Vaccination coverage $(12-39,40-59, \geq 60$ years $)$ & Description \\
\hline $90 \%$ coverage & $90 \%, 90 \%, 90 \%$ & Assuming $90 \%$ of eligible people got vaccinated \\
\hline High coverage & $75 \%, 80 \%, 90 \%$ & $\begin{array}{l}\text { Corresponding to the sum of people who are willing to be vaccinated and } \\
\text { those who cannot decide yet }\end{array}$ \\
\hline Intermediate coverage & $60 \%, 70 \%, 85 \%$ & Intermediate scenario between High and Low coverages \\
\hline Low coverage & $45 \%, 60 \%, 80 \%$ & Corresponding to a proportion of people who are willing to be vaccinated \\
\hline No vaccination & $0 \%, 0 \%, 0 \%$ & Scenario without vaccination \\
\hline $\begin{array}{l}\text { Vaccine efficacy } \\
\text { scenario }\end{array}$ & $\begin{array}{l}\text { Efficacy to prevent infection, severe illness, } \\
\text { death, and to decrease infectivity from patients } \\
\text { with a breakthrough infection }\end{array}$ & Description \\
\hline Very effective & $90 \%, 95 \%, 95 \%, 50 \%$ & $\begin{array}{l}\text { Data from clinical trials and real-world data for the original strain and the } \\
\text { Alpha variant }\end{array}$ \\
\hline Effective & $70 \%, 90 \%, 90 \%, 25 \%$ & Data for the Delta variant (the evidence is not yet sufficient) \\
\hline $\begin{array}{l}\text { Transmission pattern } \\
\text { scenario }\end{array}$ & & Description \\
\hline Heterogeneous & & $\begin{array}{l}\text { Transmissions from people aged } 20-59 \text { years and transmissions within the } \\
\text { same age group are higher than the other transmission pairs }\end{array}$ \\
\hline Homogeneous & & Transmission frequencies are the same among age groups \\
\hline
\end{tabular}

*Details of the scenarios and information sources are described in Table S1 in the Online Supplementary Document.

A computer script for the simulations is available at GitHub (https://github.com/yukifuruse1217/COVID_simulation_japan).

\section{RESULTS}

Assuming the basic reproduction number of SARS-CoV-2 was 5.0, the vaccines were very effective, and the transmission was heterogeneous among age groups (Table 1), the cumulative number of COVID-19 deaths in one season (150 days) reached 50000 in the high vaccination coverage scenario when all NPI restrictions were lifted (Figure 2, Panel A). Without any restrictions, the death count surpassed 200000

\section{A Very effective vaccines}

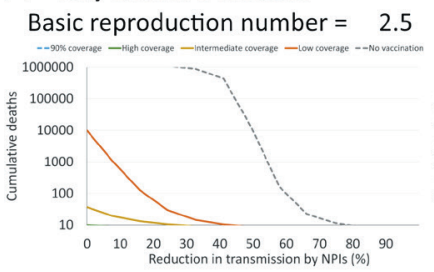

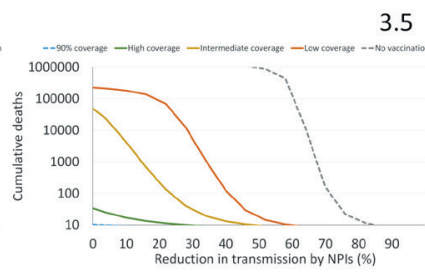

3.5

3.5

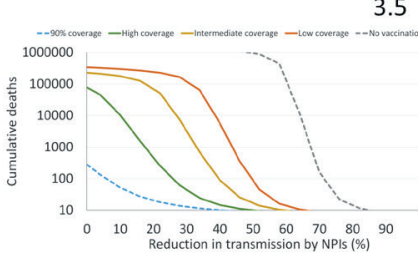

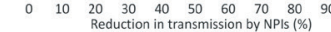

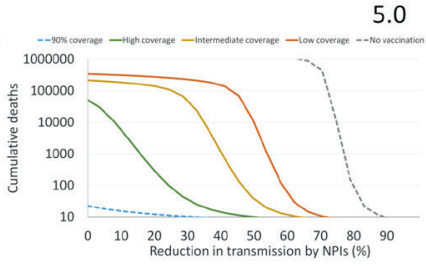

5.0

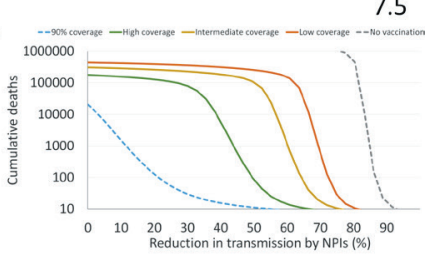

5.0
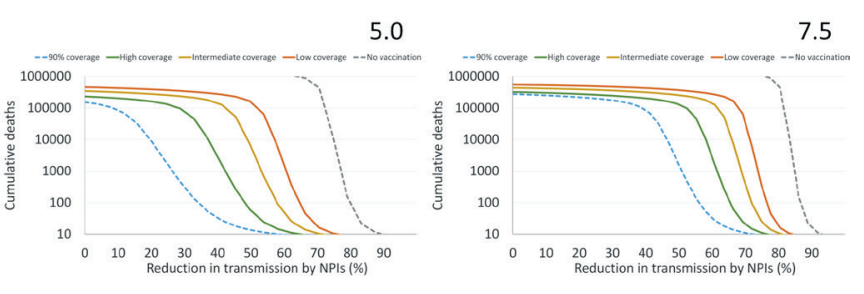

\section{B Effective vaccines}

Basic reproduction number $=2.5$

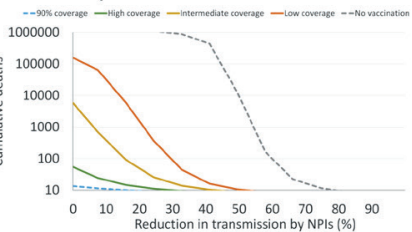

Figure 2. Cumulative numbers of COVID-19 deaths by simulation. Cumulative numbers of deaths by vaccination coverage, basic reproduction number, and vaccine efficacy in the simulation for 150 days are shown. Panel A is for very effective vaccines, and Panel B is for effective vaccines. The $\mathrm{x}$-axis denotes the degree of transmission reduction due to nonpharmaceutical intervention restrictions. The $\mathrm{y}$-axis is in a logarithmic scale. 
in the intermediate and low vaccination coverage scenarios. For comparison, the annual number of excess deaths due to influenza in Japan was approximately 10000 [27]. If we sustained the modified lifestyle observed in 2020, which could reduce transmissions by 40\% (Figure S1 in the Online Supplementary Document), the death count would be $<10000$ in the high and intermediate vaccination coverage scenarios (Figure 2, Panel A).

Most infections occurred in children and young adults in all three vaccination coverage scenarios (Figure 3, Panels A and C). However, most deaths were observed among older adults (Figure 3, Panels B and D), even though we assumed that the vaccination coverage rate was especially high for the age group (Table 1).
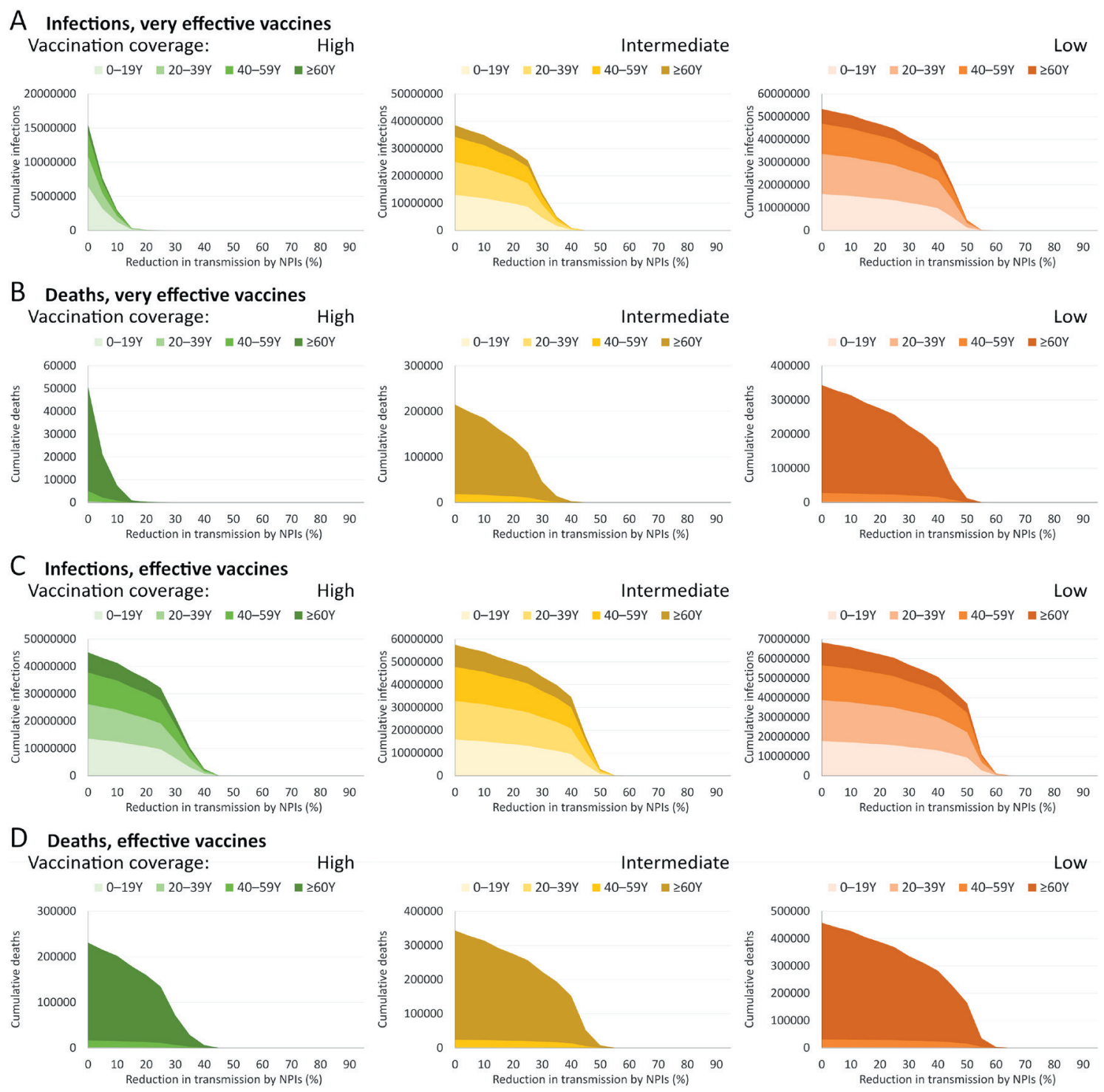

Figure 3. Cumulative numbers of COVID-19 infections and deaths by age group. Cumulative numbers of infections (A, C) and deaths (B, D) in the simulation for 150 days are shown by age group. The vaccination coverage (in three colors) and the vaccine efficacy (panels A and B for very effective vaccines; and panels C and D for effective vaccines) were subject to change. The basic reproduction number was set to 5.0. The $\mathrm{x}$-axis denotes the degree of transmission reduction due to nonpharmaceutical intervention restrictions. The y-axis is in a linear scale.

If the basic reproduction number was 2.5, corresponding to the original strain, or 3.5, corresponding to the Alpha variant of SARS-CoV-2 (Table 1), we would be able to relax restrictions from the 2020 baseline (ie, $40 \%$ ) even in the low vaccination coverage scenario (Figure 2, Panel A). On the other hand, we would keep requiring strict measures, such as those under a state of emergency, if the reproduction number of circulating viruses was 7.5 and the vaccination coverage was not high. 
As expected, the outcome would worsen if the vaccines were somewhat effective but not very effective (Table 1). With decreased vaccine efficacy for viruses with a basic reproduction number of 5.0 , the cumulative number of COVID-19 deaths would be 230000 in the high vaccination coverage scenario if we lifted all NPI restrictions (Figure 2, Panel B). With very effective vaccines, most infections and deaths occurred in unvaccinated people (Figure 4, Panels A and B). In contrast, breakthrough infections among vaccinated people would account for a larger proportion of infected people and deaths in the high vaccination coverage scenario where the vaccines had decreased efficacy (Figure 4, Panels C and D). Still, incidence and mortality rates were consistently low for vaccinated people compared with unvaccinated people (Figure 5).

\section{A Infections, very effective vaccines}
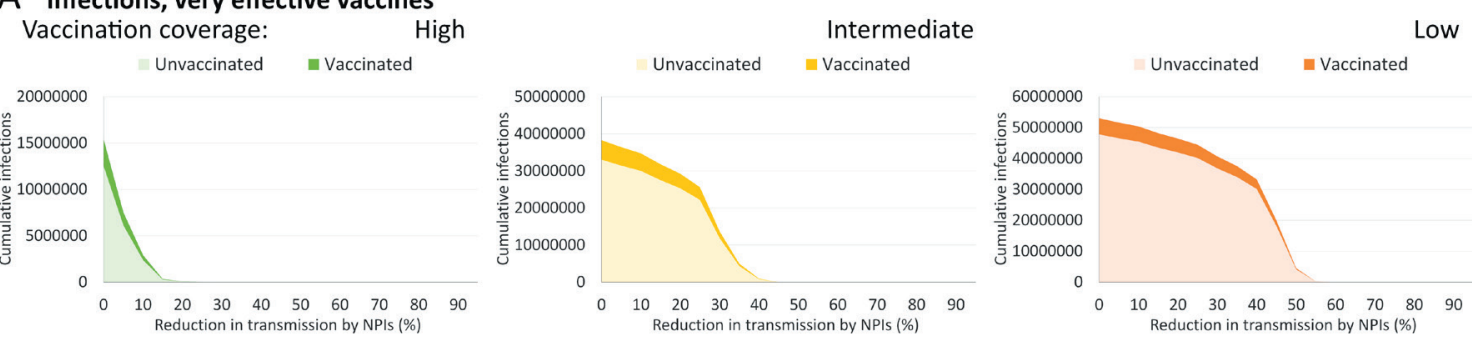

$B$ Deaths, very effective vaccines
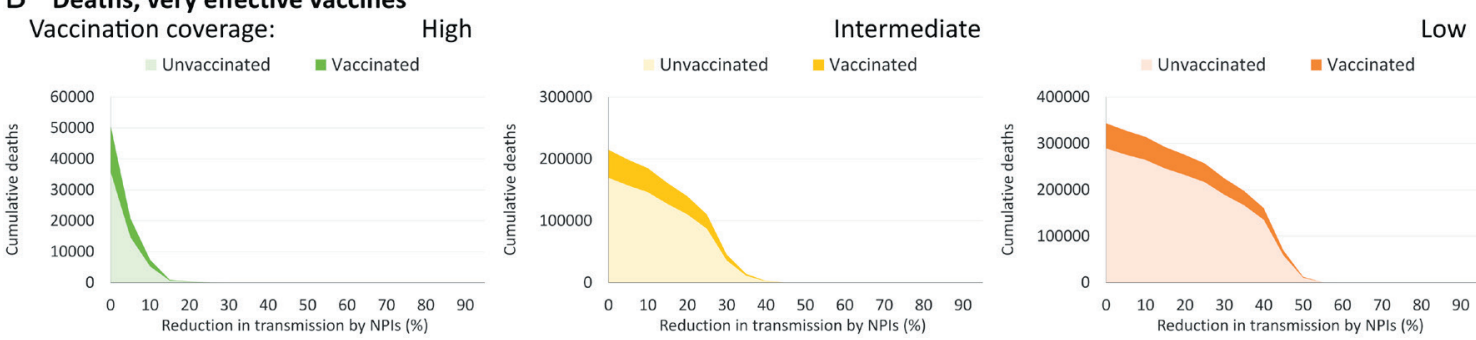

C Infections, effective vaccines
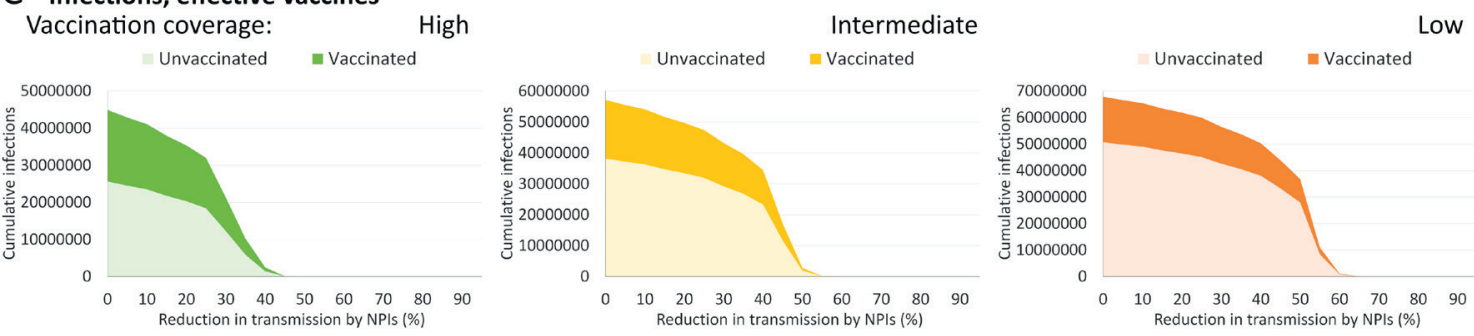

\section{Deaths, effective vaccines}
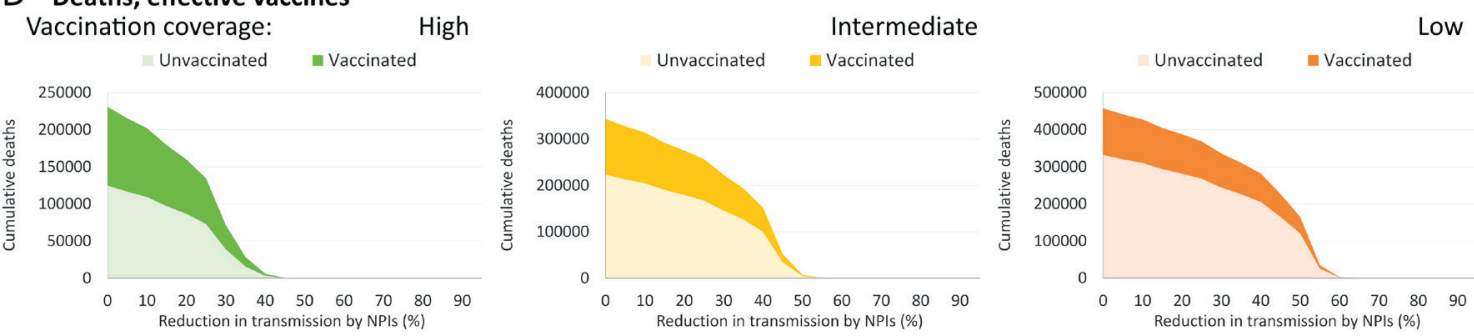

Figure 4. Cumulative numbers of COVID-19 infections and deaths by vaccination status. Cumulative numbers of infections (A, C) and deaths (B, D) in the simulation for 150 days are shown by vaccination status. The vaccination coverage (in three colors) and the vaccine efficacy (panels A and B for very effective vaccines; and panels $C$ and $D$ for effective vaccines) were subject to change. The basic reproduction number was set to 5.0. The $\mathrm{x}$-axis denotes the degree of transmission reduction due to nonpharmaceutical intervention restrictions. The y-axis is in a linear scale.

Suppose we achieved $~ 90 \%$ vaccination coverage for all age groups, as is the case for measles in many countries including Japan [28]. In that case, we could dramatically suppress the number of deaths due to COVID-19 (Figure 1, Panels A and B). The assumption of homogeneous viral transmission patterns increased the impact of the disease (Figure S2 in the Online Supplementary Document). The results of better control by herd immunity in a population with heterogeneous transmission patterns agree with the findings of a previous study [29]. 


\section{A Infections, very effective vaccines} Vaccination coverage: Unvaccinated -Vaccinated

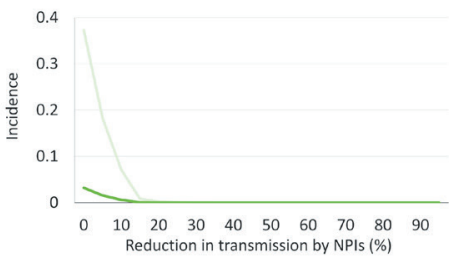

B Deaths, very effective vaccines Vaccination coverage:

Unvaccinated -Vaccinated

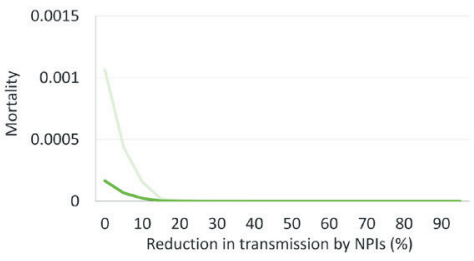

C Infections, effective vaccines Vaccination coverage:

Unvaccinated -Vaccinated

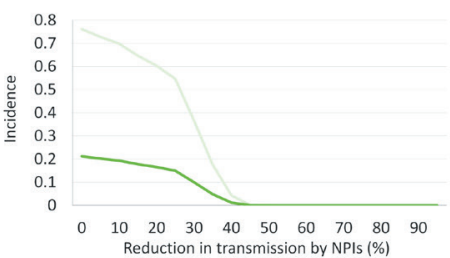

D Deaths, effective vaccines

Vaccination coverage: High Unvaccinated -Vaccinated

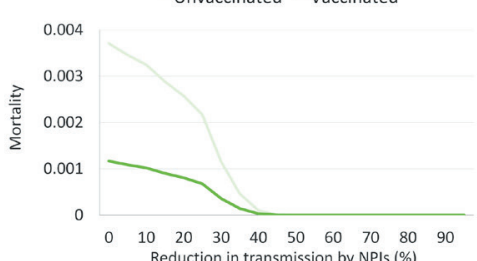

Intermediate

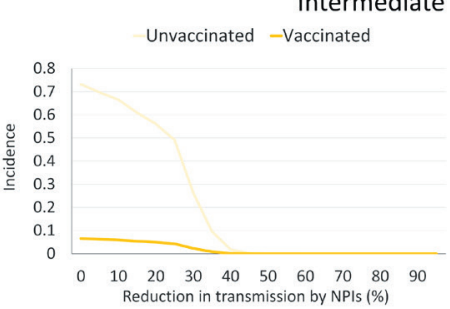

Intermediate

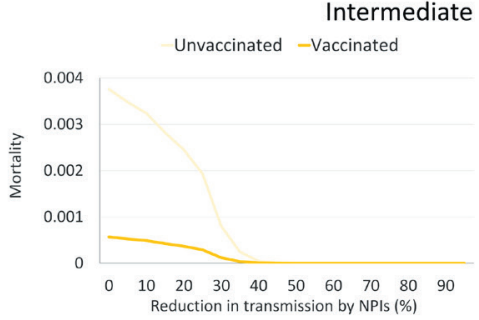

Intermediate
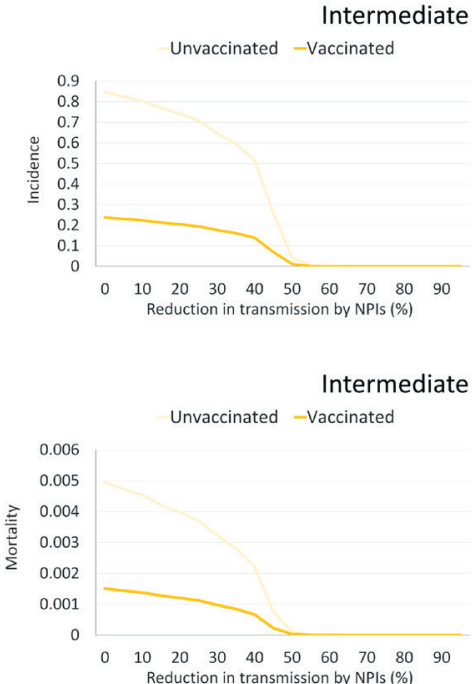
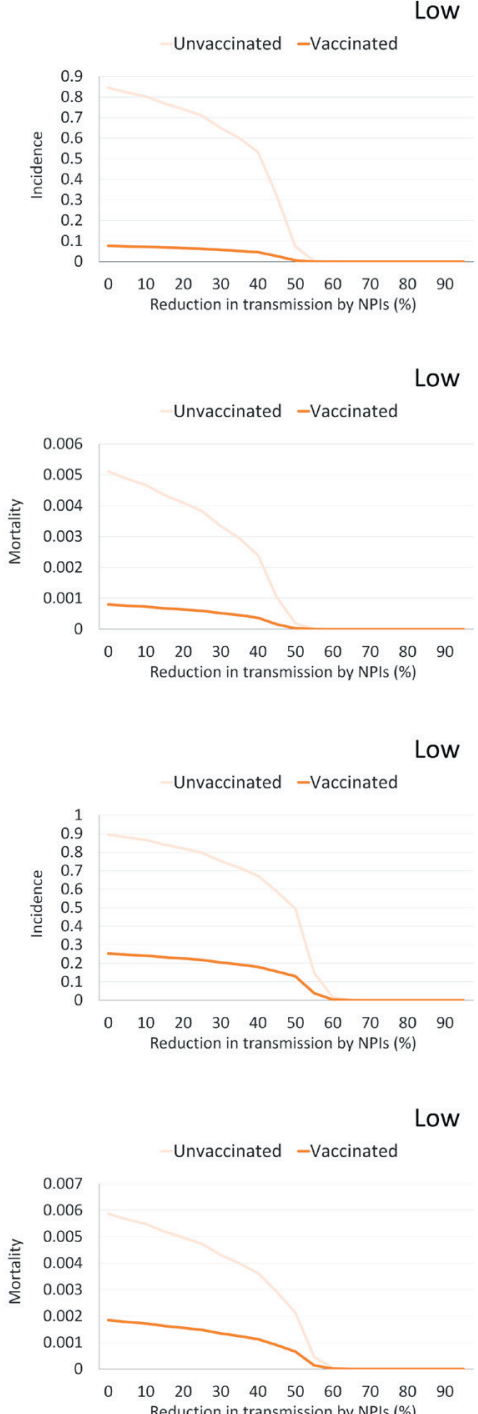

Figure 5. Incidence and mortality of COVID-19 per vaccination status. Cumulative numbers of infections (A, C) and deaths (B, D) in the simulation for 150 days were divided by the total number of either vaccinated or unvaccinated people to calculate incidence and mortality rates by vaccination status. The vaccination coverage (in three colors) and the vaccine efficacy (panels A and B for very effective vaccines; and panels $C$ and D for effective vaccines) were subject to change. The basic reproduction number was set to 5.0. The x-axis denotes the degree of transmission reduction due to nonpharmaceutical intervention restrictions. The y-axis is in a linear scale.

We finally analyzed the temporal dynamics of future COVID-19 epidemic scenarios in Japan with the first assumption: the basic reproduction number of circulating viruses was 5.0 , the vaccines were very effective, and the transmission was heterogeneous among age groups. We assumed that we would keep NPI restrictions as in 2020 (ie, 40\% reduction in transmission) or halve the restrictions from 2020 (ie, 20\% reduction in transmission) as a baseline. Moreover, we implemented a state of emergency, which can reduce transmissions by $70 \%$ (Figure S1 in the Online Supplementary Document), for 60 days when the number of patients with severe illness requiring mechanical ventilation, intensive care unit (ICU) admission, or extracorporeal membrane oxygenation surpassed 2000. In Japan, the highest number of such severe COVID-19 cases recorded by August 2021 was 2000. This indicator corresponds to the burden on health systems such as the occupancy of ICU beds. We did not take seasonal effects, including meteorological factors and holiday seasons, into account in our simulation. Voluntary, temporary changes in people's behavior without strict measures that affect transmission dynamics were also not considered.

An epidemic would not take off in the high vaccination coverage scenario with very effective vaccines and a $40 \%$ reduction in transmission as a baseline (Figure 6, Panel A). The intermediate vaccination coverage scenar- 


\section{A $40 \%$ transmission reduction as a baseline with very effective vaccines}
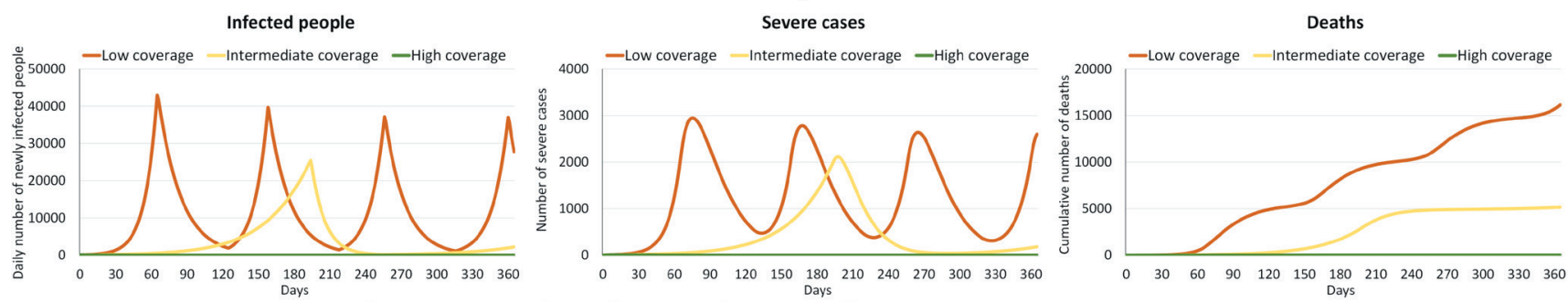

\section{B $20 \%$ transmission reduction as a baseline with very effective vaccines}
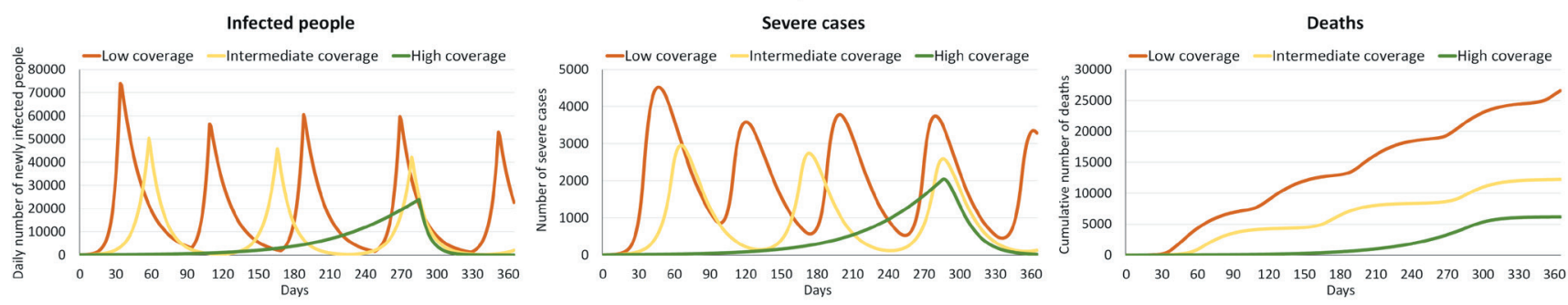

\section{C $40 \%$ transmission reduction as a baseline with effective vaccines}
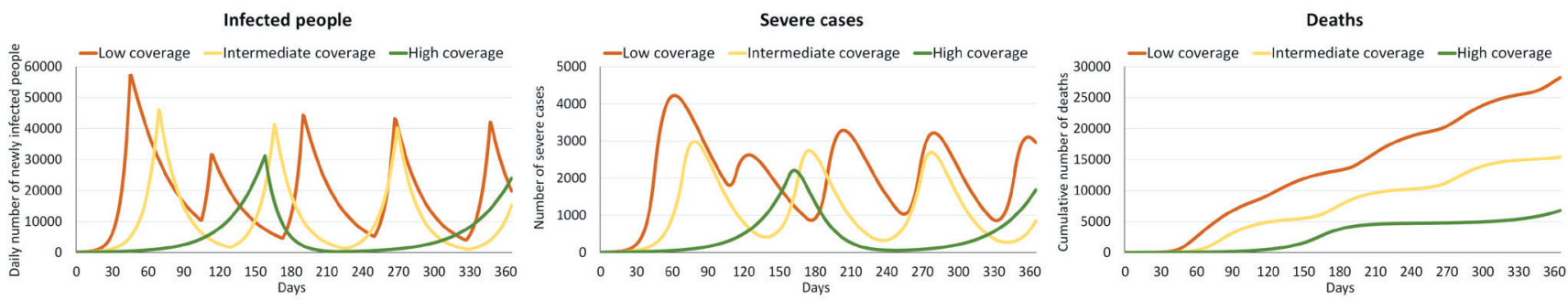

\section{D $20 \%$ transmission reduction as a baseline with effective vaccines}
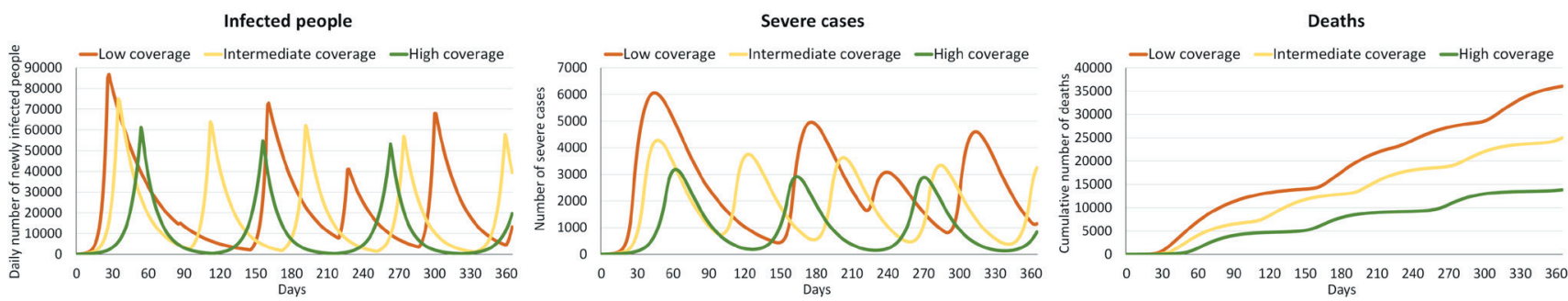

Figure 6. Temporal transmission dynamics of COVID-19 with a baseline transmission reduction and implementation of strict measures. The temporal dynamics of the numbers of newly infected people, severe cases, and cumulative deaths are shown. In the simulation, we assumed that transmission was reduced by $40 \%$ (A, C) or 20\% (B, D) as baseline nonpharmaceutical intervention restrictions. The vaccination coverage (lines in three colors) and the vaccine efficacy (panels A and B for very effective vaccines; and panels $C$ and $D$ for effective vaccines) were subject to change. Strict measures reducing transmissions by $70 \%$ were implemented for 60 days when severe cases surpassed 2000.

io required one-time implementation of strict measures. And, the low vaccination coverage scenario resulted in periodic implementation (several times in a year) of strict measures. If we relaxed our baseline restrictions from $40 \%$ to $20 \%$, repetitive implementation of strict measures would be essential to control the epidemic in both the intermediate and low vaccination coverage scenarios (Figure 6, Panel B).

One-time implementation of strict measures was enough to keep the COVID-19 epidemic under control in the high vaccination coverage scenario with a $20 \%$ transmission reduction baseline with very effective vaccines (Figure 6, Panel B) and in the high vaccination coverage scenario with a $40 \%$ reduction baseline with vaccines of somewhat decreased efficacy (Figure 6, Panel C). However, relaxing restrictions to 20\% would result in the occasional implementation of strict measures even in the high vaccination coverage scenario where vaccine efficacy was not optimal (Figure 6, Panel D). 


\section{DISCUSSION}

The results of our simulation study suggest that high vaccination coverage is important to control the COVID-19 epidemic. We can even lift or relax NPI restrictions when vaccination coverage and vaccine efficacy are high enough. However, this optimistic projection can be easily dashed when vaccination coverage is low, vaccine efficacy is insufficient, or circulating viruses are more transmissible than expected. A similar conclusion was reached by another simulation study based on situations in France [30]. Although we performed this study in the context of Japan, the results must have significant implications for other countries as well.

We did anticipate the need for periodic implementation of strict measures from the beginning of the COVID-19 pandemic [31-33]. However, we made the assumption when we did not yet have effective vaccines. With the development of effective vaccines, we hoped to eliminate the virus and return to normalcy (Figure 2, Panel A) $[34,35]$. The emergence of more transmissible variants and the possible reduction in vaccine efficacy have changed our perceptions of the disease.

The efficacy of vaccines could be even lower than our assumptions for the Delta and future variants [17,3638]. Breakthrough infections among vaccinated people impose a serious concern as we have detected a substantial difference between very effective and effective vaccine scenarios in this study. We have to keep trying to increase vaccination coverage. It should be noted that the high proportion of vaccinated people in outbreaks does not mean vaccines are ineffective $[39,40]$. We showed that it could happen when vaccination coverage is high but the efficacy is not perfect but still good (Figures 4 and 5). We may have to administer booster shots and renew the components of vaccines to deal with immunity waning and keep up with emerging variants [41-43].

Although we did not explicitly consider the effect of waning immunity in our model, decreased vaccine efficacy against the Delta variant may have covered the point to some extent $[20,44,45]$. Since this study investigated the situation where all people who are willing to be vaccinated had been already fully vaccinated, we did not consider the time-varying, increasing proportion of vaccination coverage or partial protection by a single dose of vaccination in the simulation.

This study has several limitations. Most of the parameters used in this study were empirical ones obtained from data in Japan. However, there are several uncertainties and points we did not consider in the model. These include the difference in transmissibility from asymptomatic and symptomatic patients, the difference in vaccination efficacy among age groups, and the difference in the effectiveness of NPI restrictions among different populations. Because most of the reported epidemiological parameters such as the basic reproduction number of SARS-CoV-2 was calculated not separating asymptomatic and symptomatic patients [14-16], we regard combining them in a single compartment in our model as reasonable. Although the decreased vaccine efficacy in older adults was reported, its degree varies among studies $[8,10,46]$. It was difficult to set parameters to define the age group-specific vaccine efficacy in the present study. While the effect of NPI restrictions on people's mobility and contact pattern may differ by age group [47], it remains unclear how that would affect the transmission efficiency and the course of the epidemic. To develop better models, vigilant surveillance and further research are essential.

We assessed the impact of COVID-19 mainly by the number of deaths in this study. However, several remedies have already been developed, and others are currently under development $[48,49]$. They would reduce the disease's fatality rate in the future. The burden on health systems was assessed by the number of severe cases in this study. Although the number of hospitalized patients might also be of interest for evaluating the burden, we could not take that into account. That was because the strategy about who to be hospitalized is different among prefectures in Japan. Almost all symptomatic cases including mild ones are hospitalized in some prefectures, while only patients requiring oxygen administration and patients with very high risk for developing severe illness are hospitalized in other prefectures. Furthermore, the effect of the sequela of COVID-19, dubbed "Long COVID," and the economic impact of NPIs and their indirect effects on public health, including suicide, should be considered. The inclusion of stochasticity in the model is another way for further exploration in future studies.

\section{CONCLUSIONS}

In conclusion, we should carefully explore a manageable degree of restrictions and their relaxation. The importance of the combination of vaccine rollout and NPIs has been shown in other studies as well [50,51]. We will have to keep bracing for occasional surges of the disease leading to strict measures, such as those under a 
state of emergency. Such strategies are essential even after most eligible people had been vaccinated. Although we assumed in our simulations that universal NPI restrictions would reduce transmissions, the use of vaccination certificates and negative test certificates may potentially help relax some restrictions. However, we need to address the relevant ethical issues involved [52].

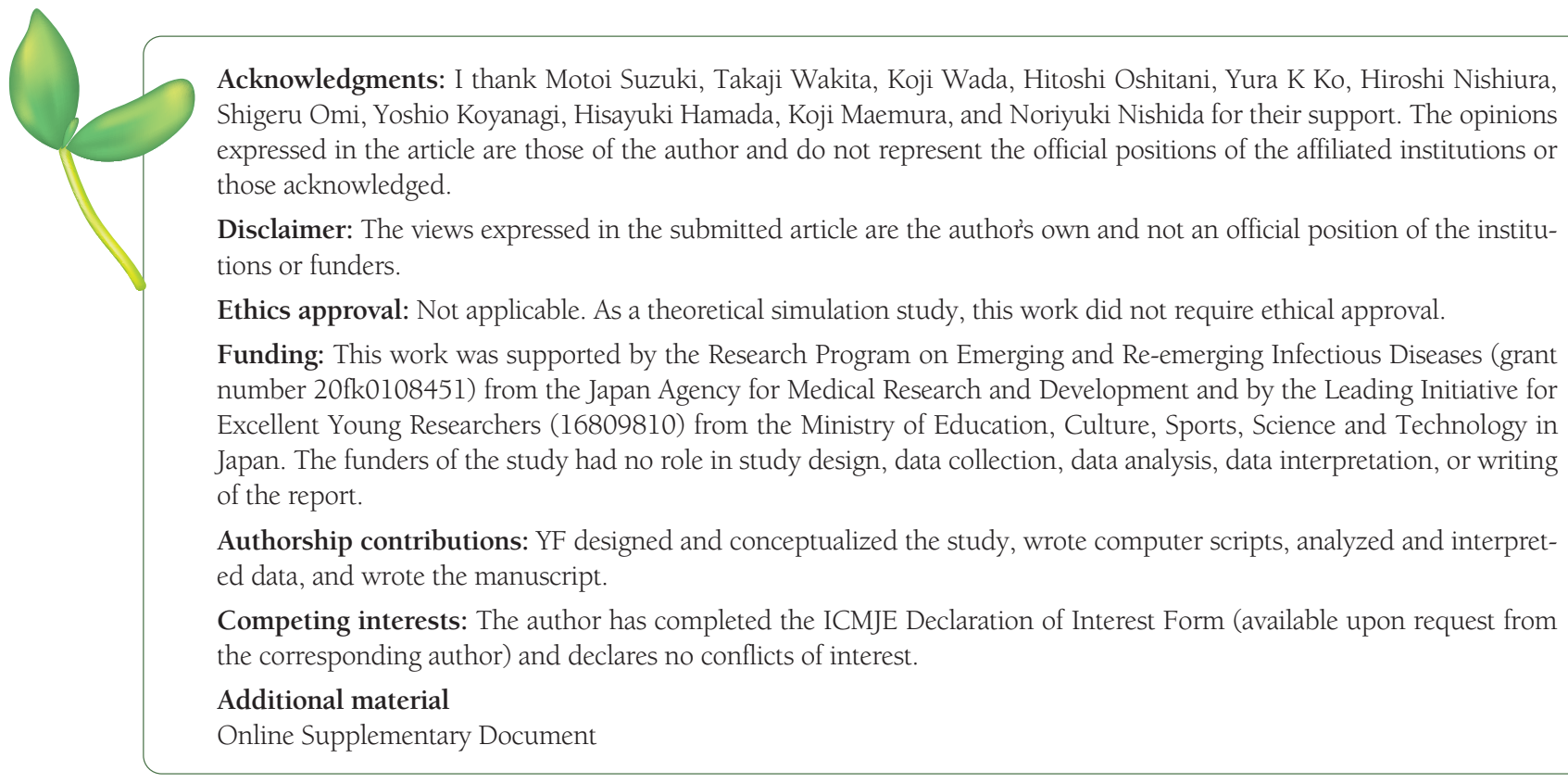

1 Furuse Y, Ko YK, Saito M, Shobugawa Y, Jindai K, Saito T, et al. Epidemiology of covid-19 outbreak in Japan, from January-March 2020. Jpn J Infect Dis. 2020;73:391-3. Medline:32350228 doi:10.7883/yoken.JJID.2020.271

2 Cowling BJ, Ali ST, Ng TWY, Tsang TK, Li JCM, Fong MW, et al. Impact assessment of non-pharmaceutical interventions against coronavirus disease 2019 and influenza in Hong Kong: an observational study. Lancet Public Health. 2020;5:e279-88. Medline:32311320 doi:10.1016/S2468-2667(20)30090-6

3 Li Y, Campbell H, Kulkarni D, Harpur A, Nundy M, Wang X, et al. The temporal association of introducing and lifting non-pharmaceutical interventions with the time-varying reproduction number (R) of SARS-CoV-2: a modelling study across 131 countries. Lancet Infect Dis. 2021;21:193-202. Medline:33729915 doi:10.1016/S1473-3099(20)30785-4

4 Karako K, Song P, Chen Y, Tang W, Kokudo N. Overview of the characteristics of and responses to the three waves of COVID-19 in Japan during 2020-2021. Biosci Trends. 2021;15:1-8. Medline:33518668 doi:10.5582/bst.2021.01019

5 Angel Y, Spitzer A, Henig O, Saiag E, Sprecher E, Padova H, et al. Association Between Vaccination With BNT162b2 and Incidence of Symptomatic and Asymptomatic SARS-CoV-2 Infections Among Health Care Workers. JAMA. 2021;325:2457-65. Medline:33956048 doi:10.1001/jama.2021.7152

6 Dagan N, Barda N, Kepten E, Miron O, Perchik S, Katz MA, et al. BNT162b2 mRNA Covid-19 Vaccine in a Nationwide Mass Vaccination Setting. N Engl J Med. 2021;384:1412-23. Medline:33626250 doi:10.1056/NEJMoa2101765

7 Tang L, Hijano DR, Gaur AH, Geiger TL, Neufeld EJ, Hoffman JM, et al. Asymptomatic and Symptomatic SARS-CoV-2 Infections After BNT162b2 Vaccination in a Routinely Screened Workforce. JAMA. 2021;325:2500-2. Medline:33956050 doi:10.1001/ jama.2021.6564

8 Baden LR, El Sahly HM, Essink B, Kotloff K, Frey S, Novak R, et al. Efficacy and Safety of the mRNA-1273 SARS-CoV-2 Vaccine. N Engl J Med. 2021;384:403-16. Medline:33378609 doi:10.1056/NEJMoa2035389

9 Tenforde MW. Effectiveness of Pfizer-BioNTech and Moderna Vaccines Against COVID-19 Among Hospitalized Adults Aged $\geq 65$ Years — United States, January-March 2021. MMWR Morb Mortal Wkly Rep. 2021;70:674-9. Medline:33956782 doi:10.15585/mmwr.mm7018el

10 Polack FP, Thomas SJ, Kitchin N, Absalon J, Gurtman A, Lockhart S, et al. Safety and Efficacy of the BNT162b2 mRNA Covid-19 Vaccine. N Engl J Med. 2020;383:2603-15. Medline:33301246 doi:10.1056/NEJMoa2034577

11 Ministry of Health Labour and Welfare (Japan). National number of COVID-19 cases and vaccination coverage among older adults and healthcare workers [in Japanese]. 2021. Available: https:/www.mhlw.go.jp/content/10900000/000796743.pdf. Accessed: 12 August 2021.

12 Roser M. Our World in Data. 2014. Available: https://ourworldindata.org. Accessed: 12 August 2021.

13 Ito K, Piantham C, Nishiura H. Predicted dominance of variant Delta of SARS-CoV-2 before Tokyo Olympic Games, Japan, July 2021. Euro Surveill. 2021;26:2100570. Medline:34240695 doi:10.2807/1560-7917.ES.2021.26.27.2100570

14 Li Q, Guan X, Wu P, Wang X, Zhou L, Tong Y, et al. Early Transmission Dynamics in Wuhan, China, of Novel Coronavirus-Infected Pneumonia. N Engl J Med. 2020;382:1199-207. Medline:31995857 doi:10.1056/NEJMoa2001316 
$15 \mathrm{Wu}$ JT, Leung K, Leung GM. Nowcasting and forecasting the potential domestic and international spread of the 2019-nCoV outbreak originating in Wuhan, China: a modelling study. Lancet. 2020;395:689-97. Medline:32014114 doi:10.1016/S01406736(20)30260-9

16 Campbell F, Archer B, Laurenson-Schafer H, Jinnai Y, Konings F, Batra N, et al. Increased transmissibility and global spread of SARS-CoV-2 variants of concern as at June 2021. Euro Surveill. 2021;26:2100509. Medline:34142653 doi:10.2807/15607917.ES.2021.26.24.2100509

17 Ministry of Health (Israel). Explanation About the Effectiveness of the Vaccine for Coronavirus in Israel. 2021. Available: https:// www.gov.il/en/departments/news/06072021-04. Accessed: 12 August 2021.

18 Riley S, Eales O, Haw D, Wang H, Walters CE, Ainslie KEC, et al. REACT-1 round 13 interim report: acceleration of SARS-CoV-2 Delta epidemic in the community in England during late June and early July 2021. medRxiv. 2021;2021.07.08.21260185.

19 Lopez Bernal J, Andrews N, Gower C, Gallagher E, Simmons R, Thelwall S, et al. Effectiveness of Covid-19 Vaccines against the B.1.617.2 (Delta) Variant. N Engl J Med. 2021;385:585-94. Medline:34289274 doi:10.1056/NEJMoa2108891

20 Puranik A, Lenehan PJ, Silvert E, Niesen MJM, Corchado-Garcia J, O'Horo JC, et al. Comparison of two highly-effective mRNA vaccines for COVID-19 during periods of Alpha and Delta variant prevalence. medRxiv. 2021;2021.08.06.21261707.

21 Sheikh A, McMenamin J, Taylor B, Robertson C. SARS-CoV-2 Delta VOC in Scotland: demographics, risk of hospital admission, and vaccine effectiveness. Lancet. 2021;397:2461-2. Medline:34139198 doi:10.1016/S0140-6736(21)01358-1

22 Twohig KA, Nyberg T, Zaidi A, Thelwall S, Sinnathamby MA, Aliabadi S, et al. Hospital admission and emergency care attendance risk for SARS-CoV-2 delta (B.1.617.2) compared with alpha (B.1.1.7) variants of concern: a cohort study. Lancet Infect Dis. 2021;S1473-3099(21)00475-8. Medline:34461056 doi:10.1016/S1473-3099(21)00475-8

23 BuzzFeed. Sixty percent of people are willing to get vaccinated in Japan [in Japanese]. 2021. Available: https://www.buzzfeed. com/jp/naokoiwanaga/covid-19-vaccine-chousa. Accessed: 12 August 2021.

24 Harris RJ, Hall JA, Zaidi A, Andrews NJ, Dunbar JK, Dabrera G. Effect of Vaccination on Household Transmission of SARSCoV-2 in England. N Engl J Med. 2021;385:759-60. Medline:34161702 doi:10.1056/NEJMc2107717

25 Shah ASV, Gribben C, Bishop J, Hanlon P, Caldwell D, Wood R, et al. Effect of Vaccination on Transmission of SARS-CoV-2. N Engl J Med. 2021. Online ahead of print. Medline:34496200 doi:10.1056/NEJMc2106757

26 Eyre DW, Taylor D, Purver M, Chapman D, Fowler T, Pouwels KB, et al. The impact of SARS-CoV-2 vaccination on Alpha Eamp; Delta variant transmission. medRxiv. 2021;2021.09.28.21264260.

27 Sugawara T, Ohkusa Y, Sugawara T, Ohkusa Y. Comparison of Models for Excess Mortality of Influenza Applied to Japan. J Biosci Med. 2019;7:13-23. doi:10.4236/jbm.2019.76002

28 GBD 2020, Release 1, Vaccine Coverage Collaborators. Measuring routine childhood vaccination coverage in 204 countries and territories, 1980-2019: a systematic analysis for the Global Burden of Disease Study 2020, Release 1. Lancet. 2021;398:50321. Medline:34273291 doi:10.1016/S0140-6736(21)00984-3

29 Britton T, Ball F, Trapman P. A mathematical model reveals the influence of population heterogeneity on herd immunity to SARS-CoV-2. Science. 2020;369:846-9. Medline:32576668 doi:10.1126/science.abc6810

30 Tran Kiem C, Massonnaud CR, Levy-Bruhl D, Poletto C, Colizza V, Bosetti P, et al. A modelling study investigating short and medium-term challenges for COVID-19 vaccination: From prioritisation to the relaxation of measures. EClinicalMedicine. 2021;38:101001. Medline:34278284 doi:10.1016/j.eclinm.2021.101001

31 Kissler SM, Tedijanto C, Goldstein E, Grad YH, Lipsitch M. Projecting the transmission dynamics of SARS-CoV-2 through the postpandemic period. Science. 2020;368:860-8. Medline:32291278 doi:10.1126/science.abb5793

32 Ferguson NM, Laydon D, Nedjati-Gilani G, Imai N, Ainslie K, Baguelin M, et al. Impact of non-pharmaceutical interventions (NPIs) to reduce COVID-19 mortality and healthcare demand. Imperial.Ac.Uk. 2020. Available: https://www.imperial.ac.uk/ mrc-global-infectious-disease-analysis/covid-19/report-9-impact-of-npis-on-covid-19. Accessed: 16 October 2021.

33 López L, Rodó X. The end of social confinement and COVID-19 re-emergence risk. Nat Hum Behav. 2020;4:746-55. Medline:32572175 doi:10.1038/s41562-020-0908-8

34 Furuse Y, Oshitani H. Viruses That Can and Cannot Coexist With Humans and the Future of SARS-CoV-2. Front Microbiol. 2020;11:583252. Medline:33042101 doi:10.3389/fmicb.2020.583252

35 Kofman A, Kantor R, Adashi EY. Potential COVID-19 Endgame Scenarios: Eradication, Elimination, Cohabitation, or Conflagration? JAMA. 2021;326:303-4. Medline:34236382 doi:10.1001/jama.2021.11042

36 Tang P, Hasan MR, Chemaitelly H. YASSINE HM, Benslimane F, Khatib HA Al, et al. BNT162b2 and mRNA-1273 COVID-19 vaccine effectiveness against the Delta (B.1.617.2) variant in Qatar. medRxiv. 2021;2021.08.11.21261885.

37 Liu Y, Arase N, Kishikawa J, Hirose M, Li S, Tada A, et al. The SARS-CoV-2 Delta variant is poised to acquire complete resistance to wild-type spike vaccines. bioRxiv. 2021;2021.08.22.457114.

38 Uriu K, Kimura I, Shirakawa K, Takaori-Kondo A, Nakada T, Kaneda A, et al. Ineffective neutralization of the SARS-CoV-2 Mu variant by convalescent and vaccine sera. bioRxiv. 2021;2021.09.06.459005.

39 Brown CM. Outbreak of SARS-CoV-2 Infections, Including COVID-19 Vaccine Breakthrough Infections, Associated with Large Public Gatherings - Barnstable County, Massachusetts, July 2021. MMWR Morb Mortal Wkly Rep. 2021;70:1059-62. Medline:34351882 doi:10.15585/mmwr.mm7031e2

40 Williams SV, Vusirikala A, Ladhani SN, De Olano EFR, Iyanger N, Aiano F, et al. An outbreak caused by the SARS-CoV-2 Delta (B.1.617.2) variant in a care home after partial vaccination with a single dose of the COVID-19 vaccine Vaxzevria, London, England, April 2021. Euro Surveill. 2021;26:2100626. Medline:34240699 doi:10.2807/1560-7917.ES.2021.26.27.2100626

41 Patalon T, Gazit S, Pitzer VE, Prunas O, Warren JL, Weinberger DM. Short Term Reduction in the Odds of Testing Positive for SARSCoV-2; a Comparison Between Two Doses and Three doses of the BNT162b2 Vaccine. medRxiv. 2021;2021.08.29.21262792. 
42 Bar-On YM, Goldberg Y, Mandel M, Bodenheimer O, Freedman L, Kalkstein N, et al. Protection of BNT162b2 Vaccine Booster against Covid-19 in Israel. N Engl J Med. 2021;385:1393-400. Medline:34525275 doi:10.1056/NEJMoa2114255

43 Choi A, Koch M, Wu K, Chu L, Ma L, Hill A, et al. Safety and immunogenicity of SARS-CoV-2 variant mRNA vaccine boosters in healthy adults: an interim analysis. Nat Med. 2021. Online ahead of print. Medline:34526698 doi:10.1038/s41591021-01527-y

44 Keehner J, Horton LE, Binkin NJ, Laurent LC, Pride D, Longhurst CA, et al. Resurgence of SARS-CoV-2 Infection in a Highly Vaccinated Health System Workforce. N Engl J Med. 2021;385:1330-2. Medline:34469645 doi:10.1056/NEJMc2112981

45 Thomas SJ, Edson D. Moreira J, Kitchin N, Absalon J, Gurtman A, Lockhart S, et al. Safety and Efficacy of the BNT162b2 mRNA Covid-19 Vaccine through 6 Months. N Engl J Med. 2021. Online ahead of print. Medline:34525277 doi:10.1056/ NEJMoa2110345

46 Chodick G, Tene L, Rotem RS, Patalon T, Gazit S, Ben-Tov A, et al. The Effectiveness of the Two-Dose BNT162b2 Vaccine: Analysis of Real-World Data. Clin Infect Dis. 2021;ciab438. Medline:33999127 doi:10.1093/cid/ciab438

47 Nomura S, Tanoue Y, Yoneoka D, Gilmour S, Kawashima T, Eguchi A, et al. Mobility Patterns in Different Age Groups in Japan during the COVID-19 Pandemic: a Small Area Time Series Analysis through March 2021. J Urban Health. 2021. Medline:34379269 doi:10.1007/s11524-021-00566-7

48 Lamontagne F, Agoritsas T, MacDonald H, Leo YS, Daz J, Agarwal A, et al. A living WHO guideline on drugs for covid-19. BMJ. 2021;372:n526. Medline:33649077 doi:10.1136/bmj.n526

49 Siemieniuk RA, Bartoszko JJ, Ge L, Zeraatkar D, Izcovich A, Pardo-Hernandez H, et al. Drug treatments for covid-19: living systematic review and network meta-analysis. BMJ. 2020;370:m2980. Medline:32732190 doi:10.1136/bmj.m2980

50 Patel MD, Rosenstrom E, Ivy JS, Mayorga ME, Keskinocak P, Boyce RM, et al. Association of Simulated COVID-19 Vaccination and Nonpharmaceutical Interventions With Infections, Hospitalizations, and Mortality. JAMA Netw Open. 2021;4:e2110782. Medline:34061203 doi:10.1001/jamanetworkopen.2021.10782

51 Leung K, Wu JT, Leung GM. Effects of adjusting public health, travel, and social measures during the roll-out of COVID-19 vaccination: a modelling study. Lancet Public Health. 2021;6:e674-82. Medline:34388389 doi:10.1016/S2468-2667(21)00167-5

52 Hall MA, Studdert DM. "Vaccine Passport" Certification - Policy and Ethical Considerations. N Engl J Med. 2021;385:e32. Medline:33789006 doi:10.1056/NEJMp2104289 